CORRECTION

https://doi.org/10.1038/s41586-019-1360-7

\title{
Publisher Correction: Structure and function of Vms1 and Arb1 in RQC and mitochondrial proteome homeostasis
}

Ting Su, Toshiaki Izawa, Matthias Thoms, Yui Yamashita, Jingdong Cheng, Otto Berninghausen, F. Ulrich Hartl, Toshifumi Inada, Walter Neupert \& Roland Beckmann

Correction to: Nature https://doi.org/10.1038/s41586-019-1307-z, published online 12 June 2019.

In Fig. $2 \mathrm{~g}$ of this Letter, owing to an error during the production process, the bottom blot for 'IB: Myc' was missing. The original Letter has been corrected online. 